\title{
SOPORTE TECNOLÓGICO: EVOLUCIÓN EN EL TIEMPO, CUADRO COMPARATIVO EMPRESARIAL
}

\author{
TECHNOLOGICAL SUPPORT: EVOLUTION IN TIME, BUSINESS \\ COMPARATIVE TABLE
}

\author{
Esp. Rolando Quintero Pérez*, MSc. Avilio Villamizar Estrada** \\ MSc. Maritza del Pilar Sánchez Delgado** \\ * Universidad de Pamplona, Pamplona, Norte de Santander, Colombia. \\ *Centro de Investigación Aplicada y Desarrollo en Tecnologías de Información (CIADTI). \\ **Grupo de Investigación en Ciencias Computacionales (CICOM). \\ E-mail: \{rquintero, aviliove, pilas\}@ unipamplona.edu.co.
}

\begin{abstract}
In the execution of the project Management Model Applying Balanced Scorecard in the Process Of Technological Support of the Center for Applied Research and Information Technology Development - CIADTI - from the University of Pamplona, this document presents the analysis of the state of art and the existing structures related to the evolution of technological support, innovation and the way companies provide the service in order to identify the factors which influence the continuous improvement of the process from the CIADTI University of Pamplona.
\end{abstract}

Keywords: Technological support, service, process, model.

Resumen: En la ejecución del Proyecto Modelo de Gestión Aplicando Balanced Scorecard en el Proceso de Soporte Tecnológico del CIADTI Universidad de Pamplona, el presente documento presenta un análisis del estado del arte y estructuras existentes acerca de la evolución del soporte tecnológico, la innovación y forma en que las empresas prestan el servicio para identificar los factores que inciden en el mejoramiento continuo.

Palabras clave: Soporte tecnológico, servicio, proceso, modelo.

\section{INTRODUCCIÓN}

Como base estructural del diseño del modelo de gestión para el proceso de soporte tecnológico del CIADTI Universidad de Pamplona, se hizo necesario indagar sobre la evolución en la prestación del servicio y los dispositivos que intervienen para su desarrollo, así como las innovaciones y estructuras funcionales de diferentes empresas incluyendo la de Soporte Tecnológico del CIADTI para establecer un comparativo entre ellas y socavar dentro de sus funcionamientos variables que sirvan de insumo en la construcción del modelo. El proyecto de investigación nace de la necesidad de encontrar en la prestación del servicio de soporte algo más allá de la solución a un problema, brindar una asesoría y/o realizar una implantación o actualización.

El presente artículo establece una línea de tiempo en la evolución de los dispositivos eléctricos y electrónicos base fundamental en la prestación del servicio de soporte tecnológico, así como paralelamente una línea de tiempo de la evolución del mismo lo cual refleja entre las dos un estado comparativo y puntos críticos de partida en el mejoramiento de dicho proceso. Gracias a lo anterior, las empresas partiendo de sus necesidades y estructuras organizativas han establecido procedimientos de innovación y funcionamiento 
dando a conocer variables que definen criterios de medición que dan un punto de partida de forma en que se presta el servicio de soporte tecnológico a cada uno de sus clientes.

\section{EVOLUCIÓN DEL SOPORTE TECNOLÓGICO}

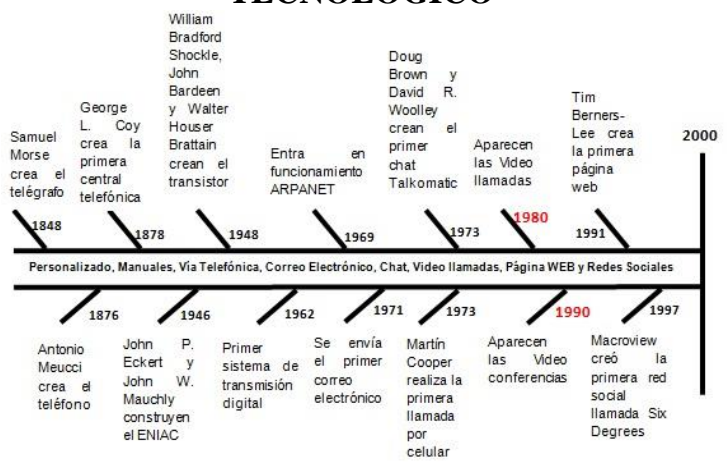

Fig. 1. Evolución tecnológica del servicio de soporte

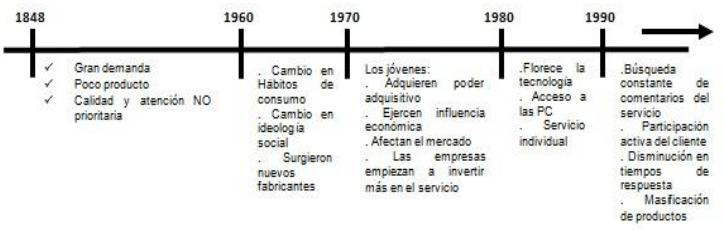

Fig. 2. Evolución del servicio de soporte tecnológico

El servicio al cliente ha evolucionado a lo largo del tiempo debido a los diferentes acontecimientos y necesidades de la época en que se ha encontrado el ser humano. En 1848 gracias a la necesidad de ser humano de comunicarse sin tener ningún tipo de barreras el inquieto inventor Samuel Morse crea el telégrafo, en 1876 Antonio Meucci crea el teléfono y en 1878 George L. Coy crea la primera central telefónica con lo que la barrera espacio tiempo en las comunicaciones empezó a ser derrumbada por el ser humano.

Desde entonces y hasta poco antes de los años 50 la atención y la calidad en los servicios que se prestaban no están dentro de la prioridad de los inventores y empresas nacientes y mantenían encerrado al cliente dado a la mucha demanda y poca oferta de productos en el mercado. En 1946 John P. Eckert y John W. Mauchly construyen una de las primeras computadoras de propósito general llamada ENIAC y en 1948 se revoluciona la electrónica con la creación del transistor llevando a las empresas a producir dispositivos más usables, portables en mayor cantidad para el ser humano.
En los años 60 y 70, los hábitos de consumo aumentaron considerablemente y la ideología social de los jóvenes y su mejoramiento de su poder adquisitivo generaron nuevas estructuras de cambio apareciendo nuevos fabricantes que rápidamente filtraron los mercados y generaron competencia hacia la floreciente demanda de nuevos productos. Fue entonces que en 1962 se crea el primer sistema de trasmisión digital y en 1969 entra en funcionamiento ARPANET, en 1971 se crea el primer correo electrónico y en 1973 se produce la primera conversación mediante chat y teléfono celular convirtiéndose esto en la base de las redes de comunicación actuales y haciendo de estas décadas las más brillantes para el comienzo del mejoramiento en la prestación del servicio al cliente.

Entre los años 80 y 90 la tecnología tiene la mayor revolución y coloco al alcance de todo el mundo un dispositivo que cambiaría por completo la historia del manejo de la prestación del servicio al cliente, la computadora. Gracias a lo anterior aparecen las primeras video llamadas y video conferencias llegando a recibir un servicio individual, personalizado y en cierto grado privado. De los años 90 en adelante con la creación de las páginas WEB y las redes sociales se generó una nueva historia en la prestación del servicio ya que hoy en día los tiempos de respuesta han disminuido y la masificación de productos han hecho del servicio al cliente una variable muy significativa a la hora de adquirir un producto.

Por lo anterior, la prestación del servicio al cliente evolucionó no solo por la masificación de los productos sino por la evolución de la electrónica y los sistemas de computación ya que ellos son hoy en día el bastión fundamental para la prestación de un bien servicio.

\section{INNOVACIÓN PARA MEJORAR EL SERVICIO}

Karl Albrecht (1990) en su libro "La revolución del servicio" expresa en la categoría del servicio como arte lo siguiente: "estas son las empresas que están instaladas en la mente del consumidor por su calidad en deleitar al cliente. Tienen una consagración obsesiva por la calidad de los servicios que brindan" y concluye diciendo: "sus Operadores tienen una forma de "ser y pensar" que los distingue de los demás. Pero no por lo que dicen, sino por lo que su equipo "hace",". 


\subsection{Estrategias para mejorar el servicio al cliente}

Tabla 1: Estrategias para mejorar

\begin{tabular}{|c|c|}
\hline & GENERALES \\
\hline $\begin{array}{l}\text { Definición } \\
\text { de servicio }\end{array}$ & $\begin{array}{l}\text { Es necesario brindar al cliente } \\
\text { además de un servicio básico, un } \\
\text { cuidando especial en el trato y la } \\
\text { información que se le otorga. }\end{array}$ \\
\hline $\begin{array}{l}\text { Saber quién } \\
\text { es el cliente }\end{array}$ & $\begin{array}{l}\checkmark \text { No es un extraño, es la persona } \\
\text { más importante para la } \\
\text { organización. } \\
\checkmark \quad \text { La persona de quien depende } \\
\text { la organización. } \\
\checkmark \text { Es nuestro objetivo de trabajo, } \\
\text { por lo tanto, el cliente jamás } \\
\text { interrumpe. }\end{array}$ \\
\hline $\begin{array}{l}\text { Actitud } \\
\text { amistosa }\end{array}$ & $\begin{array}{ll}\checkmark & \text { Se debe servir por el placer de } \\
& \text { servir. } \\
\checkmark & \text { Nunca sustituir } \\
& \text { conveniencia por el servicio. } \\
\checkmark & \text { Sentir una reclamación como } \\
& \text { una solicitud servicio. } \\
\checkmark & \text { Decir la verdad al cliente. } \\
\checkmark & \text { Atender a los clientes sin } \\
& \text { necesidad de escalar al jefe de } \\
\text { área. } \\
\checkmark & \text { Usted es el único responsable } \\
& \text { de su trabajo. } \\
\checkmark & \text { El placer de los negocios y del } \\
\text { trabajo proviene del servir y } \\
\text { no de las ventas. }\end{array}$ \\
\hline
\end{tabular}

\section{ORGANIZACIONALES}

Encuestas de opinión
Permiten conocer y medir el grado de satisfacción de los clientes respecto a la prestación del servicio como base de una estrategia de intervención para la elaboración de planes de mejoramiento a corto, mediano y largo plazo.

Planes de Una vez se ha establecido el acción para estado actual de la prestación del mejorar el servicio se debe desarrollar un servicio. plan de trabajo con metas y fechas concretas teniendo en cuenta los siguientes actividades y tareas:
Actividades que debe realizar la empresa:

$\checkmark$ Evaluar y monitorear la calidad del servicio.

$\checkmark$ Diseñar los procedimientos de atención al cliente.

$\checkmark$ Establecimiento de normas de servicio.

$\checkmark$ Capacitación al personal.

$\checkmark$ Programas de incentivos.

$\checkmark$ Mejora de estos procesos.

Tareas que debe realizar el trabajador:

$\checkmark$ Participar en grupos de mejora.

$\checkmark$ Asistir a la capacitación.

$\checkmark$ Aplicar los procedimientos.

Elaborar un El programa de entrenamiento programa de entrenamien to para su personal debe estar dirigido a las directivas y trabajadores de la organización ya que cada uno desde su rol debe actuar de manera sinérgica con el otro para lograr una satisfacción plena del cliente con una óptima prestación del servicio.

Habilidades que deben tener en cuenta el personal directivo:

$\checkmark$ Conceptos básicos de servicio al cliente.

$\checkmark$ Diseño de instrumentos de evaluación.

$\checkmark$ Diseño de estrategias organizacionales.

$\checkmark$ Implementación de programas de mejora continua.

$\checkmark$ Diseño de normas y procedimientos.

$\checkmark$ Manejo de personal orientado hacia el servicio.

Habilidades que deben tener en cuenta el trabajador:

$\checkmark$ Conceptos básicos de servicio al cliente.

$\checkmark$ Estrategias para obtener un buen servicio.

$\checkmark$ Estrategias individuales de mejoramiento.

Establecer Es una herramienta de gestión grupos de cuya misión es proponer 


\begin{tabular}{|c|c|}
\hline \multirow[t]{2}{*}{$\begin{array}{l}\text { mejora } \\
\text { continua }\end{array}$} & $\begin{array}{l}\text { soluciones a procesos o } \\
\text { actividades con una metodología } \\
\text { definida. }\end{array}$ \\
\hline & $\begin{array}{l}\text { Sus principales características } \\
\text { son: } \\
\checkmark\end{array}$ \\
\hline
\end{tabular}

Establecer normas de servicio

La organización debe crear como política las normas de servicio las cuales deben contener las reglas, procedimientos de atención y conductas aplicables en la atención a clientes.

Diseñar un Es muy importante que la programa organización diseñe como integral de estrategia de mejoramiento un reconocimie plan de recompensas ya que éstas ntos.

son poderosos incentivos para mejorar la satisfacción del empleado, su desempeño y por ende la satisfacción del cliente.

Algunos tipos de reconocimientos son:

$\checkmark$ Económicos.

$\checkmark$ Promoción.

$\checkmark$ Días compensatorios.

$\checkmark$ Menciones de honor.

\section{INDIVIDUALES}

\begin{abstract}
Auto El trabajador debe dedicar tiempo aprendizaje para fortalecer los aspectos en los que tiene falencias al prestar el servicio.
\end{abstract}

Manejo del El trabajador debe manejar tiempo efectivamente el tiempo tanto con el cliente como en el desempeño de otras actividades dentro de la organización.

Conocer El trabajador debe estudiar cada cada vez proceso y procedimiento más el realizado por la organización para cliente y sus poder entregar soluciones procesos optimas a las dificultades del cliente.

\section{ESTRUCTURA DE SERVICIO DE SOPORTE TECNOLÓGICO}

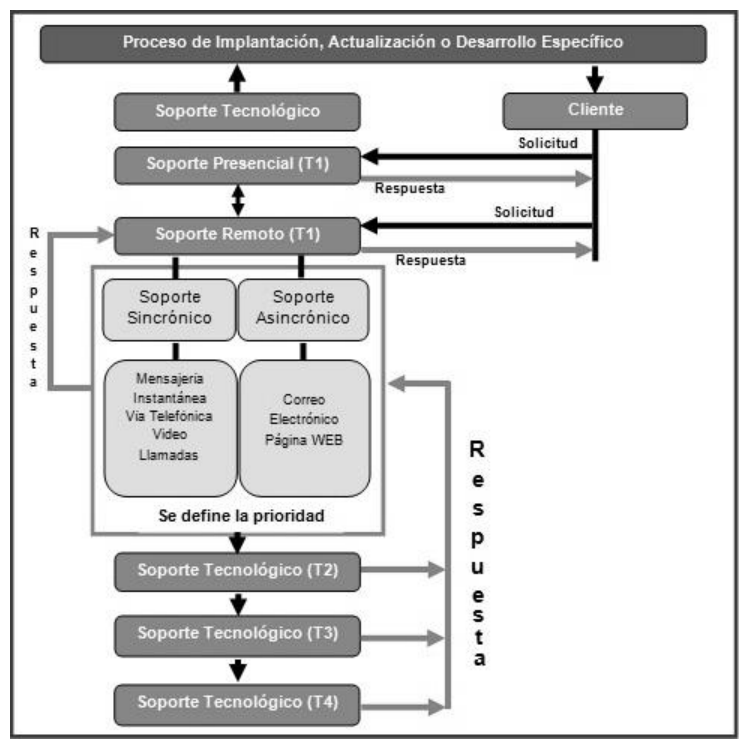

Fig. 3. Estructura de servicio de soporte tecnológico

Durante el proceso de soporte tecnológico el cliente puede presentar dos tipos de acciones: solicitudes al soporte presencial y solicitudes al soporte remoto. En primera instancia, se realiza durante el proceso de implantación, actualización o desarrollo específico que se esté implementando en la organización. La segunda instancia ocurre una vez se entregue funcional y con los usuarios capacitados el sistema de información. En ambos casos, la solicitud la recibe un soporte de nivel 1.

Cuando el soporte es remoto, éste se puede realizar de dos formas de comunicación: sincrónico usando herramientas como: la mensajería instantánea, vía telefónica y video llamadas o asincrónico usando herramientas como: correo electrónico y página WEB (Jaime Guzmán-Luna et al., 2013, Jaime Guzmán-Luna et al., 2014).

En cualquiera de los dos casos la solicitud es tomada por un profesional de soporte de nivel 1 . 
Estando en ese punto se define la prioridad y la complejidad de la solicitud dando respuesta prontamente si la complejidad es crítica. En caso de que la complejidad sea mayor la solicitud es trasladada a un profesional de soporte de nivel 2 y así sucesivamente hasta llegar a un nivel 4 que es el equipo interdisciplinario de desarrollo.

Finalmente, la respuesta es trasladada al cliente mediante el profesional de soporte de nivel 1 que recibió la solicitud.

\section{METODOLOGÍAS DE APLICACIÓN DEL SERVICIO}

\subsection{Caso Hewlett Packard}

La empresa Hewlett Packard en su enlace http://welcome.hp.com/country/us/en/prodserv/soft ware/eda/pdf/HP_Exstream_Support_Guide_Octob er_2012_Latin_American_Spanish.pdf presenta la forma en que soporta técnicamente al cliente que adquiere el software HP Exstream. Adicionalmente del enlace https://support.hp.com/co-es que es la página oficial de soporte tecnológico de los diferentes productos y servicios de HP extraemos la información pertinente para nuestro caso de investigación.

\begin{tabular}{|c|c|c|c|c|c|c|c|c|c|}
\hline \multicolumn{10}{|c|}{ Soporte Técnico del Software HP Exstream } \\
\hline \multicolumn{2}{|c|}{ Soporte Presencial } & \multicolumn{5}{|c|}{ Sincrónico } & \multicolumn{3}{|c|}{ Asincrónico } \\
\hline \multicolumn{2}{|c|}{ si } & \multicolumn{2}{|c|}{$\begin{array}{l}\text { Mensajeria } \\
\text { Instantanea }\end{array}$} & $\begin{array}{c}\text { Via } \\
\text { Telefónica } \\
\text { (a) }\end{array}$ & \multicolumn{2}{|c|}{$\begin{array}{l}\text { Video } \\
\text { Llamadas }\end{array}$} & \multicolumn{2}{|r|}{$\begin{array}{l}\text { Correo } \\
\text { Electrónico }\end{array}$} & $\begin{array}{c}\text { Página } \\
\text { WEe }\end{array}$ \\
\hline \multicolumn{4}{|c|}{$\begin{array}{l}\text { Niveles de Soporte } \\
\text { ryindados }\end{array}$} & $\begin{array}{c}\text { S1 } \\
\text { Apertura y } \\
\text { Cierre de } \\
\text { lncidentes }\end{array}$ & \multicolumn{2}{|c|}{$\begin{array}{l}\text { Tiempo de } \\
\text { Prestación del } \\
\text { servicio }\end{array}$} & \multicolumn{2}{|c|}{ SI } & $\begin{array}{l}\text { Tiempos de } \\
\text { Respevesta }\end{array}$ \\
\hline Nivel & $\frac{N_{2}}{N_{2}}$ & $\frac{\text { Nivel }}{3}$ & $\begin{array}{c}\text { Nivel } \\
4\end{array}$ & \multirow{2}{*}{ Incidentes } & \multirow{2}{*}{\multicolumn{2}{|c|}{$\begin{array}{l}24 \text { horas } \\
\text { De } 8: 00 \text { am a } \\
5: 00 \text { ph horas } \\
\text { y diabs hobiales } \\
\text { locales }\end{array}$}} & \multirow{2}{*}{\multicolumn{2}{|c|}{$\begin{array}{l}\text { Gravedad } 1=\text { Cntica } \\
\text { Gravedad } 2=\text { Seria } \\
\text { Gravedad } 3=\text { Media } \\
\text { Gravedad } 4=\text { Baia }\end{array}$}} & \multirow{2}{*}{$\begin{array}{c}2 \text { horas } \\
6 \text { horas hábiles } \\
8 \text { horas hábiles } \\
1 \text { día hábil }\end{array}$} \\
\hline s! & sn & sin & sin & & & & & & \\
\hline $\begin{array}{l}\text { Varios } \\
\text { Idiomas }\end{array}$ & $\begin{array}{l}\text { Vincule } \\
\text { Rede } \\
\text { Sociak }\end{array}$ & & $\begin{array}{l}\text { Permite } \\
\text { unimiento a }\end{array}$ & $\begin{array}{l}\text { Estructura } \\
\begin{array}{c}\text { Manejo de de } \\
\text { de Actualiza }\end{array}\end{array}$ & $\begin{array}{l}\text { la Pág } \\
\text { cargas } \\
\text { cones }\end{array}$ & $\begin{array}{l}\text { a WEE } \\
\text { Desc } \\
\text { Manu }\end{array}$ & $\begin{array}{l}\text { ade } \\
\text { es de }\end{array}$ & $\begin{array}{l}\text { Sitio de } \\
\text { Preguntas } \\
\end{array}$ & $\begin{array}{l}\text { Soporte Técnico en } \\
\text { linea }\end{array}$ \\
\hline S1 & $\frac{\text { Social }}{\text { SI }}$ & & & $\overline{s !}$ & & & & Frecuentes & \\
\hline
\end{tabular}

Fig. 4. Soporte técnico del software HP Exstream. Fuente: Hewlett Packard

\subsection{Caso International Business Machines IBM}

La empresa International Business Machines (IBM) en su enlace http://www935.ibm.com/services/us/igs/pdf-isscontracts/mexico-7818-00.pdf presenta la forma en que soporta técnicamente al cliente que adquiere IBM ISS (Internet Security Systems) dedicada a la seguridad del software. Adicionalmente del enlace https://support.podc.sl.edst.ibm.com/support/home/ que es la página oficial de soporte tecnológico de los diferentes productos y servicios de IBM extraemos la información pertinente para nuestro caso de investigación.

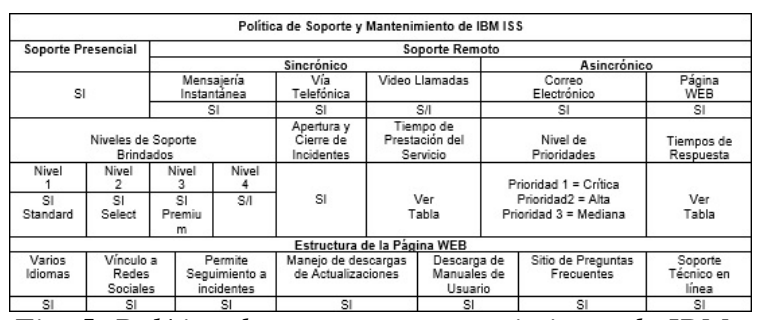

Fig. 5. Política de soporte y mantenimiento de IBM para productos y servicios. Fuente: IBM ISS

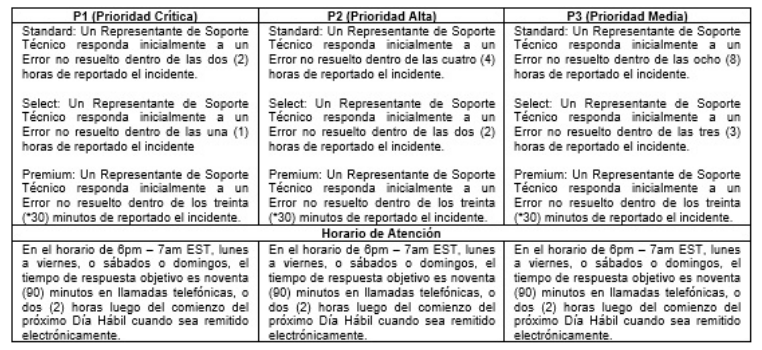

Fig. 6. Tiempo de respuesta y horario de atención. Fuente: IBM ISS

\subsection{Caso Microsoft Office 365}

La empresa Microsoft Office 365 en su enlace https://technet.microsoft.com/es-co/library/office365-support.aspx presenta la forma en que soporta técnicamente al cliente que adquiere uno de los productos y/o servicios de donde extraemos la información pertinente para nuestro caso de investigación.

\begin{tabular}{|c|c|c|c|c|c|c|c|c|c|}
\hline \multicolumn{10}{|c|}{ Microsoft Office 365} \\
\hline \multicolumn{2}{|c|}{ Soporte Presencial } & \multicolumn{6}{|c|}{ Soporte Remoto } & \multicolumn{2}{|c|}{ Asincrónico } \\
\hline \multicolumn{2}{|c|}{ si } & \multicolumn{2}{|c|}{$\begin{array}{l}\text { Mensajeria } \\
\text { Instantánea }\end{array}$} & $\begin{array}{l}\text { Via } \\
\text { Telefónica }\end{array}$ & Lan & & \multicolumn{2}{|r|}{$\begin{array}{l}\text { Correo } \\
\text { Electrónioo }\end{array}$} & $\begin{array}{l}\text { Página } \\
\text { WeB }\end{array}$ \\
\hline \multicolumn{4}{|c|}{$\begin{array}{l}\text { Niveles de Soporte } \\
\text { Brindados }\end{array}$} & $\begin{array}{l}\text { Aperturay } \\
\text { Cierre de } \\
\text { Incidentes }\end{array}$ & $\begin{array}{l}\text { Tien } \\
\text { Presta } \\
\text { Se }\end{array}$ & $\begin{array}{l}\text { ode } \\
\text { ond del } \\
\text { icio }\end{array}$ & & $\begin{array}{l}\text { Nivel de } \\
\text { Prioridades }\end{array}$ & $\begin{array}{l}\text { Tiempos se } \\
\text { Respouesta }\end{array}$ \\
\hline $\begin{array}{l}\text { Nivel } \\
1\end{array}$ & $\begin{array}{l}\text { Nivel } \\
2\end{array}$ & $\begin{array}{c}\text { Nivel } \\
3\end{array}$ & $\begin{array}{l}\text { Nivel } \\
4\end{array}$ & \multirow[t]{2}{*}{$\mathrm{Sl}$} & \multirow{2}{*}{\multicolumn{2}{|c|}{$\begin{array}{c}\text { Ver } \\
\text { Tabla }\end{array}$}} & \multirow{2}{*}{\multicolumn{2}{|c|}{ 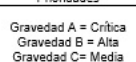 }} & \multirow{2}{*}{$\begin{array}{c}\text { Ver } \\
\text { Tablas }\end{array}$} \\
\hline SI & SI & SII & SA & & & & & & \\
\hline $\begin{array}{l}\text { Varios } \\
\text { Idiomas }\end{array}$ & $\begin{array}{l}\text { Vinculo } \\
\text { Redes } \\
\text { Sodeg }\end{array}$ & \multicolumn{2}{|c|}{$\begin{array}{c}\text { Permite } \\
\text { Segumimanto a } \\
\text { incidentes }\end{array}$} & \multicolumn{2}{|c|}{ 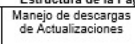 } & \multicolumn{2}{|c|}{$\begin{array}{l}\text { Descarga de } \\
\text { Manualas de } \\
\text { Usuario }\end{array}$} & $\begin{array}{c}\text { Stitio de } \\
\text { Preguntas } \\
\text { Feros }\end{array}$ & $\begin{array}{c}\text { Soporte Técnico } \\
\text { en linea }\end{array}$ \\
\hline SI & S! & & & SI & & & & 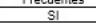 & SI \\
\hline
\end{tabular}

Fig. 7. Estructura de soporte tecnológico Microsoft Office 365. Fuente: Microsoft Office 365

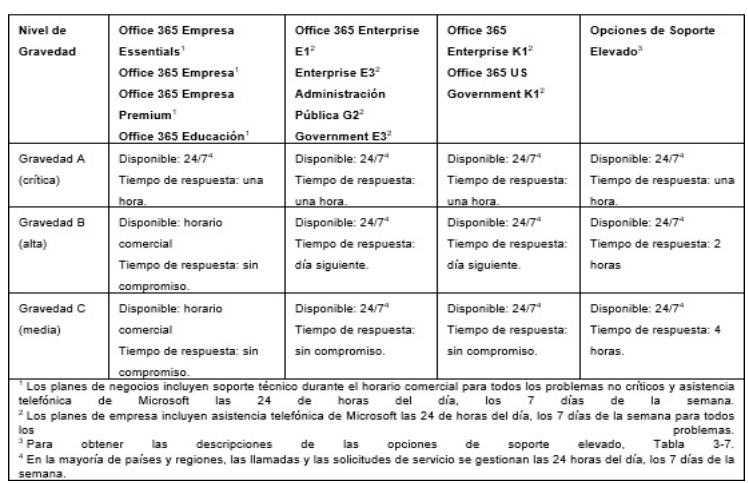

Fig. 8. Tiempo de respuesta y horario de atención. Fuente: Microsoft Office 365 


\subsection{Caso Oracle}

La empresa Oracle en su enlace https://www.oracle.com/lad/assets/software-policylad.pdf presenta la forma en que soporta técnicamente al cliente que adquiere uno de sus productos. Adicionalmente del enlace https://www.oracle.com/support/index.html que es la página oficial de soporte tecnológico de los diferentes productos y servicios de Oracle extraemos la información pertinente para nuestro caso de investigación.

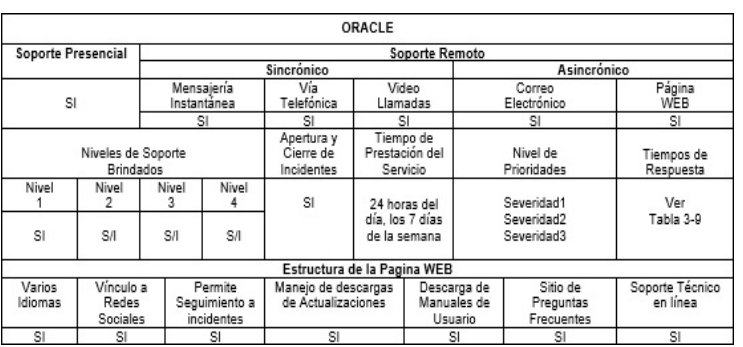

Fig. 9. Estructura de soporte tecnológico Oracle. Fuente: Oracle

\begin{tabular}{|c|c|c|c|}
\hline Nivel de Severidad & Tiempo de Respuesta' & $\begin{array}{c}\text { Tiempo de Restauración } \\
\text { Remota' }^{\prime}\end{array}$ & Tiempo de Resolución ' \\
\hline Severidad 1 & 15 minutos & 6horas & 30 días corridos \\
\hline Severidad 2 & 15 minutos & 48 horas & 30 días corridos \\
\hline Severidad 3 & NC & NIC & 180 días corridos \\
\hline $\begin{array}{l}\text { VTiempo de Respuesta: } \\
\text { primera vez. } \\
\text { v Tiempo de Restauració } \\
\text { hasta que Oracle le no } \\
\text { requieren cambios al co } \\
\text { vTiempo de Resolución: }\end{array}$ & $\begin{array}{l}\text { se ha ofrecido una s } \\
\text { programa. }\end{array}$ & $\begin{array}{l}\text { que Oracle logra el acce } \\
\text { os Tiempos de Restaur }\end{array}$ & $\begin{array}{l}\text { ta que Oracle le responde por } \\
\text { moto al programa respectivo } \\
\text { Remota no se aplican si se }\end{array}$ \\
\hline
\end{tabular}

Fig. 10. Tiempo de respuesta. Fuente: Oracle

\subsection{Caso Solutec Informática}

La empresa Solutec Informática en su enlace http://www.solutekcolombia.com/contacto/ayuda_t ecnologica.htm presenta la forma en que soporta técnicamente al cliente que adquiere uno de los productos $\mathrm{y} / \mathrm{o}$ servicios. Adicionalmente en el enlace

http://www.solutekcolombia.com/servicios_tecnolo gicos/outsourcing_tecnologico/index.htm que es la página oficial de soporte tecnológico de los diferentes productos y servicios extraemos la información pertinente para nuestro caso de investigación.

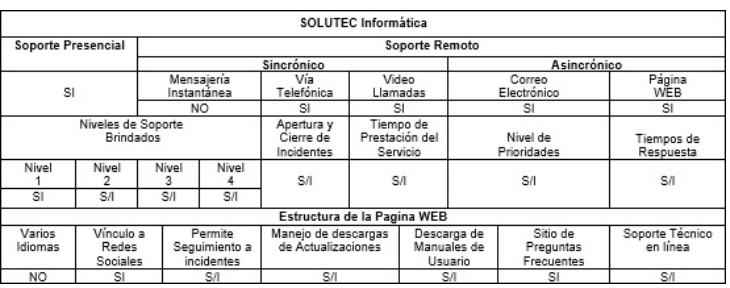

Fig. 11. Política de soporte Solutec Informática.

Fuente: Solutec Informática

\subsection{Caso CIADTI}

La unidad del CIADTI Universidad de Pamplona en su enlace:

http://www.unipamplona.edu.co/soportetecnologic $o /$ presenta la forma en que soporta técnicamente al cliente que adquiere uno de los productos y/o servicios de donde extraemos la información pertinente para nuestro caso de investigación.

\begin{tabular}{|c|c|c|c|c|c|c|c|c|c|}
\hline \multicolumn{10}{|c|}{ CIADTI } \\
\hline \multicolumn{2}{|c|}{ Soporte Presencial } & \multicolumn{8}{|c|}{ Soporte Remoto } \\
\hline \multirow{3}{*}{\multicolumn{2}{|c|}{ sI }} & \multirow{3}{*}{\multicolumn{2}{|c|}{$\begin{array}{l}\text { Mensajeria } \\
\text { Instantánea }\end{array}$}} & \multicolumn{3}{|l|}{ Sincrónico } & \multicolumn{3}{|c|}{ Asincrónico } \\
\hline & & & & $\begin{array}{c}\text { Via } \\
\text { Telefónica } \\
\text { The }\end{array}$ & & dod & & $\begin{array}{l}\text { Correo } \\
\text { Electrónico }\end{array}$ & $\begin{array}{c}\text { Página } \\
\text { WeB }\end{array}$ \\
\hline & & & & $\mathrm{SI}$ & & & & SI & Si \\
\hline \multicolumn{4}{|c|}{$\begin{array}{l}\text { Niveles de Soporte } \\
\text { Brindados }\end{array}$} & $\begin{array}{l}\text { Aperturay } \\
\text { Ciered de } \\
\text { Incidentes }\end{array}$ & $\begin{array}{l}\text { Tiem } \\
\text { Presta } \\
\text { Ser }\end{array}$ & $\begin{array}{l}\text { ofo de } \\
\text { oín del } \\
\text { icio }\end{array}$ & & $\begin{array}{l}\text { Nivel de } \\
\text { Prioridades }\end{array}$ & $\begin{array}{l}\text { Tiempos de } \\
\text { Respuesta }\end{array}$ \\
\hline $\begin{array}{c}\text { Nivel } \\
1 \\
1\end{array}$ & $\begin{array}{c}\text { Nivel } \\
2\end{array}$ & $\begin{array}{c}\text { Nivel } \\
3\end{array}$ & $\begin{array}{c}\text { Nivel } \\
4\end{array}$ & \multirow{2}{*}{ miventus } & \multirow{2}{*}{\multicolumn{2}{|c|}{$\begin{array}{c}\text { Lunes a } \\
\text { viernes de } \\
8: 00 \mathrm{am} \mathrm{a} \\
12: 00 \mathrm{~m} \mathrm{y} \mathrm{de} \\
14: 00 \mathrm{a} 18: 00 \\
\text { sábado de } \\
8: 00 \mathrm{am} \text { a } \\
12: 00 \mathrm{~m}\end{array}$}} & \multirow{2}{*}{\multicolumn{2}{|c|}{$\begin{array}{l}\text { Prioridad } 1=\text { Critica } \\
\text { Prioridad } 2=\text { Alta } \\
\text { Prioridad } 3=\text { Media } \\
\text { Prioridad } 4=\text { = Baja }\end{array}$}} & \multirow[b]{2}{*}{$\begin{array}{l}2 \text { horas hábiles } \\
8 \text { horas hábiles } \\
5 \text { dís habilies } \\
10 \text { dias hábiles }\end{array}$} \\
\hline SI & sI & SII & SI & & & & & & \\
\hline \multicolumn{10}{|c|}{$\begin{array}{c}1 \text { 12:00 m } \\
\text { Estructura de la Pagina WEB }\end{array}$} \\
\hline \begin{tabular}{|c|} 
Varios \\
Idiomas
\end{tabular} & $\begin{array}{l}\text { Vincul } \\
\text { Rede } \\
\text { Social }\end{array}$ & & $\begin{array}{l}\text { Permite } \\
\text { guimiento a } \\
\text { ncidentes }\end{array}$ & $\begin{array}{l}\text { Manejo de de } \\
\text { de Actualiza }\end{array}$ & $\begin{array}{l}\text { cargas } \\
\text { iones }\end{array}$ & $\begin{array}{l}\text { Descez: } \\
\text { Manu } \\
\text { Mas }\end{array}$ & & $\begin{array}{c}\text { Sitio de } \\
\text { Preguntas } \\
\text { Freguntes }\end{array}$ & $\begin{array}{l}\text { Soporte Técnico } \\
\text { en linea }\end{array}$ \\
\hline NO & $\frac{\text { Notili }}{\text { NO }}$ & & & NO & & & & $\begin{array}{c}\text { Frecuentes } \\
\text { NO }\end{array}$ & SI \\
\hline
\end{tabular}

Fig. 12. Estructura de soporte CIADTI. Fuente: CIADTI

\section{PRESTACIÓN DEL SERVICIO ACTUAL}

\subsection{Roles que intervienen en la prestación del servicio}

\begin{tabular}{|c|c|}
\hline \multicolumn{2}{|r|}{ SOPORTE TECNOLOGICO (CIADTI) } \\
\hline Rol & Descripción \\
\hline Subdirector de Soporte Tecnológico & $\begin{array}{l}\text { Realiza la coordinación, supervisión y seguimiento a cada uno de los equipos de soporte } \\
\text { del CIADTI, asi como a cada uno de sus analístas. }\end{array}$ \\
\hline $\begin{array}{l}\text { Analistas Soporte ACADEMUSOFT } \\
\text { IES / UNIPAMPLONA }\end{array}$ & $\begin{array}{l}\text { Encargado de la comunicación con el cliente IES / UNIPAMPLONA para ayudar a a } \\
\text { resolver inquietudes ylo problemas que se puedan presentar a los usuarios en la } \\
\text { ejecoución de sus procesos en el ambiente ACADEMUSOFT. }\end{array}$ \\
\hline $\begin{array}{l}\text { Analistas Soporte GESTASOFT } \\
\text { IES / UNIPAMPLONA }\end{array}$ & $\begin{array}{l}\text { Encargado de la comunicación con el cliente IES / UNIPAMPLONA para ayudar a a } \\
\text { resolver inquietudes ylo problemas que se puedan presentar a los usuarios en la } \\
\text { ejecoución de sus procesos en el ambiente GESTASOFT. }\end{array}$ \\
\hline $\begin{array}{l}\text { Analistas Soporte Formación } \\
\text { Continuada IES / UNIPAMPLONA }\end{array}$ & $\begin{array}{l}\text { Encargado de la comunicación con el cliente IES / UNIPAMPLONA para ayudar a a } \\
\text { resolver inquietudes ylo problemas que se puedan presentar a los usuarios en la } \\
\text { ejecoción de sus prooesos en el ambiente Formación Continuada. }\end{array}$ \\
\hline $\begin{array}{l}\text { Analistas Soporte Software } \\
\text { Especifico IES / UNIPAMPLONA }\end{array}$ & $\begin{array}{l}\text { Encargado de la comunicación con el cliente IES / UNIPAMPLONA para ayudar a a } \\
\text { resolver inquietudes ylo problemas que se puedan presentar a los usuarios en la } \\
\text { ejecoución de sus prooesos del software especifico desarrolado. }\end{array}$ \\
\hline Analistas Soporte CONCURSOS & $\begin{array}{l}\text { Encargado de la comunicación con la persona responsable del software de concursos } \\
\text { para ayudar a resolver inquietudes yo problemas que se puedan presentar a los } \\
\text { usuarios en la ejecucuión de sus procesos. }\end{array}$ \\
\hline Usuarios finales & $\begin{array}{l}\text { Personal interlocutor con el equipo de Soporte Tecnológico del CIADTI los cuales } \\
\text { reciben las capacitaciones para el manejo del producto y se encargan de reportar las } \\
\text { inquietudes yio problemas que se puedan presentar en la ejecución de sus procesos. }\end{array}$ \\
\hline
\end{tabular}

Fig. 13. Roles soporte tecnológico CIADTI

\subsection{Niveles de prioridad y tiempos de respuesta}

\begin{tabular}{|c|c|c|c|c|}
\hline \multicolumn{5}{|c|}{ SOPORTE TECNOLOGICO (CIADTI) } \\
\hline Prioridad & Impacto & Abre. & $\begin{array}{c}\text { Situación } \\
\text { Identificada }\end{array}$ & $\begin{array}{l}\text { Tiempo de } \\
\text { Respuesta }\end{array}$ \\
\hline 1 & Critica & P1 & $\begin{array}{l}\text { Fallo general o imposibilidad de realizar } \\
\text { cualquier operacion que se requiere } \\
\text { ejecutar de inmediato, ocassionado por } \\
\text { fallas de la adlicación. }\end{array}$ & $\begin{array}{l}\text { Atención inmediata, con } \\
\text { tiempo de respuesta entre } \\
\text { cero }(0) \text { y dos }(2) \text { horas } \\
\text { laborables. }\end{array}$ \\
\hline 2 & Alta & P2 & $\begin{array}{l}\text { Imposibilidad de utilización u operación } \\
\text { normal de algunas funciones conticas de } \\
\text { uso diario. ocasionado por fallas de la } \\
\text { applicasción. }\end{array}$ & $\begin{array}{l}\text { Atención prioritaria, con } \\
\text { tiempo de respuesta entre dos } \\
\text { (2) y ocho (8) horas } \\
\text { laborables. }\end{array}$ \\
\hline 3 & Media & P3 & $\begin{array}{l}\text { Imposibilidad de utilización u operación } \\
\text { nonmal de aplicaciones o funciones } \\
\text { importantes, pero no criticas, ocasionado } \\
\text { por fallas de la aplicación. }\end{array}$ & $\begin{array}{l}\text { Atención en prioridad media, } \\
\text { con tiempo de respuesta entre } \\
\text { un (1) día y cinco (5) días } \\
\text { hábiles. }\end{array}$ \\
\hline 4 & Baja & P4 & $\begin{array}{l}\text { Imposibilidad de utilización u operación } \\
\text { normal de funciones no crticicas. } \\
\text { ocasionado por fallas de la aplicación. }\end{array}$ & $\begin{array}{l}\text { Atención en prioridad baja, con } \\
\text { tiempo de respuesta entre } \\
\text { cinco (5) días y diez (10) días } \\
\text { hábiles. }\end{array}$ \\
\hline
\end{tabular}

Fig. 14. Niveles de prioridad y tiempos de respuesta. Fuente: Soporte Tecnológico CIADTI 


\subsection{Metodología de prestación del servicio}

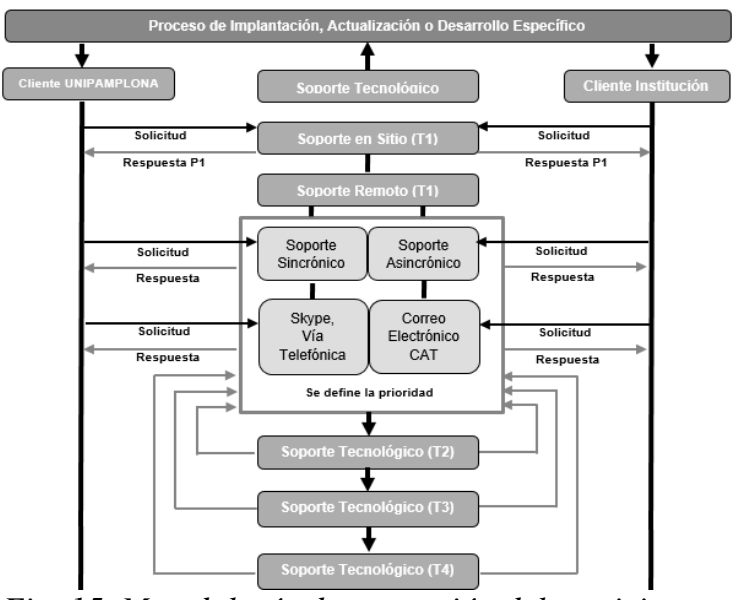

Fig. 15. Metodología de prestación del servicio

Durante el proceso de soporte tecnológico del CIADTI el cliente puede presentar dos tipos de acciones las cuales pueden provenir del cliente principal que es la Universidad de Pamplona o de un cliente de una institución externa.

Ambas solicitudes se reciben por medio de una canal presencial o un canal remoto. En primera instancia, se realiza durante el proceso de implantación, actualización o desarrollo específico que se esté realizando en la organización. La segunda instancia ocurre una vez se entregue funcional y con los usuarios capacitados el sistema de información. En ambos casos la solicitud la recibe un Analista de Soporte de nivel 1.

Cuando el soporte es remoto, éste se puede realizar e dos formas de comunicación: Sincrónico usando herramientas como: Skype y vía telefónica o asincrónico usando herramientas como: correo electrónico y CAT. En cualquiera de los dos casos la solicitud es tomada por un analista de soporte de nivel 1.

Estando en ese punto se define la prioridad de la solicitud dando respuesta de acuerdo con el tiempo de respuesta establecido para cada prioridad. En caso de que la complejidad sea mayor la solicitud es trasladada a un profesional de soporte de nivel $2 y$ en última instancia al nivel 4 que es el equipo interdisciplinario de desarrollo.

Finalmente, la respuesta es trasladada al cliente mediante el analista de soporte de nivel 1 que recibió la solicitud (María C Bonfante, Andrés Castillo, 2014).

\section{CUADRO COMPARATIVO EMPRESARIAL}

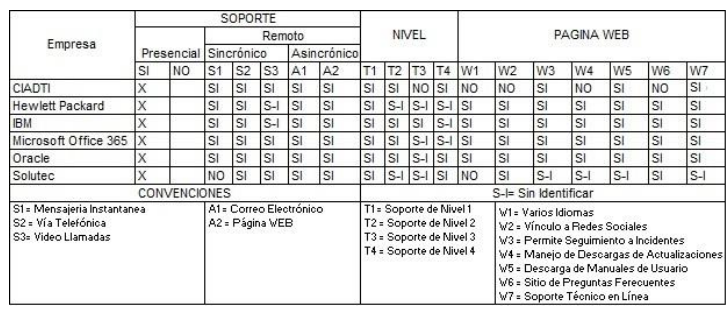

Fig. 16. Cuadro comparativo empresarial

El cuadro comparativo empresarial muestra que las seis (6) organizaciones prestan un soporte presencial ya sea en el momento de la implantación o actualización o en cada una de las instalaciones donde venden los diferentes productos y/o servicios. En cuando al soporte remoto, este proceso es soportado mediante mecanismos sincrónicos y/o asincrónicos siendo la vía telefónica, el correo electrónico y la página WEB los mecanismos predominantes para la atención a los clientes.

Dentro de la incursión de las organizaciones en ámbitos de internacionalización y globalización, las empresas multinacionales llevan la vanguardia presentando su servicio de soporte en diferentes idiomas y comunicaciones mediante comunidades y grupos en redes sociales facilitando a los clientes la interlocución no solo con los profesionales de soporte de las empresas sino también con personas que hayan adquirido los diferentes productos.

Uno de los retos en las empresas del siglo XXI es romper las barreras tecnológicas bajo los lineamientos normativos de cada país logrando establecer lenguajes universales a través del uso de las TIC ofreciendo cada día al usuario nuevas formas de usabilidad en los contenidos, mejor atención y efectividad competitiva a la hora de prestar el servicio aumentando el grado de satisfacción del cliente.

Finalmente, todas las empresas ofrecen un soporte de nivel 1 que se encarga de recibir las diferentes solicitudes de los clientes, pero en muy pocas se logra identificar el nivel de escalabilidad del trámite en caso de que el primer círculo de soporte no pueda dar una respuesta oportuna al mismo.

\section{CONCLUSIONES}

El proceso de soporte tecnológico en las organizaciones es un servicio que evolucionó con 
el tiempo y aun hoy gracias a aristas que intervienen como la competitividad, la globalización, el manejo de sistemas de información, el aumento de migrantes y nativos digitales, el fortalecimiento normativo y el sostenimiento de las empresas en el mercado globalizado regional, nacional y mundial hacen de este servicio siga evolucionando en la búsqueda constante de la satisfacción integral el cliente.

Una vez analizados los diferentes procesos de soporte en varias empresas incluyendo el del CIADTI Universidad de Pamplona se puede identificar que dicho servicio no trasciende las barreras operativas de sus procedimientos internos aun siendo éste el puente de interlocución entre los usuarios finales y la empresa prestadora del servicio impidiendo que ésta última se retroalimente y estanque su evolución.

\section{REFERENCIAS}

Zempoaltecatl Ibarra, Christian., Mendoza Ríos, Jacobo, Belmont Escamilla, L. H., Pacheco Larios, Miriam., y Zempoaltecatl Luqueno, OSCAR. (2010). Modelo de Gestión para la Atención de Incidentes a Usuarios de Servicios de Tecnologías de la Información (Tesis doctoral). Recuperado de http://tesis.ipn.mx/bitstream/handle/12345678 9/7783/C7.1452.pdf?sequence $=1 \&$ is Allowed $=$ $\mathrm{y}$

Solá, J. M. G., y María, J. (2003). Indicadores de gestión para las entidades públicas, In VIII Congresso Internacional del Clad sobre la reforma del estado y de la administración pública, Panamá (pp. 28-31).

Jaime Guzman-Luna, Luis Alfonso Lezcano Rodríguez, Sebastián A Gómez Arias (2013). Caracterización de los Elementos del Diagrama de Objetivos de KAOS a partir de Lenguaje Natural. Revista Colombiana de Tecnologías de Avanzada ISSN: 1692-7257 Volumen 1 - Número 21.

Jaime Guzmán-Luna, Ingrid-Durley Torres, Juan Felipe Alvarez (2014). Propuesta de un generador de aplicaciones educativas basadas en televisión digital usando arquitectura de cómputo en la nube. Revista Colombiana de Tecnologías de Avanzada ISSN: 1692-7257 Volumen 2 - Número 24.
Fernández, M. A. F. (2003). El control, fundamento de la gestión por procesos, Esic Editorial.

Alvear, R. (2016). Guía para la Implantación de la Aplicación Informática ACADEMUSOFT (Módulos Académico Pregrado Presencial y Gestasoft) Desarrollada Por La Universidad De Pamplona (Tesis de maestría). Universidad de Pamplona, Pamplona, Colombia.

Albrecht, K. (1990). La revolución del servicio, Legis.

Morales, J. E. A., y Vargas, J. E. (2010). Servicio al cliente, Asociación Oaxaqueña de Psicología AC.

María Claudia Bonfante, Andrés Castillo (2014). Integración de sistema multi-agente, ontologías y procesos de negocios como marco tecnológico de la estrategia "gobierno en línea”. Revista Colombiana de Tecnologías de Avanzada ISSN: 1692-7257 - Volumen 1 Número 23.

\section{SITIOS WEB}

Soporte técnico del software HP Exstream. (2012). HP Exstream Support Guide. http://welcome.hp.com/country/us/en/prodser v/software/eda/pdf/HP_Exstream_Support_G uide_October_2012_Latin_American_Spanis h.pdf

Política de Soporte y Mantenimiento de IBM para productos y Servicios. (2007). IBM ISS. INTC-7818-00 ESP Pol. http://www935.ibm.com/services/us/igs/pdf-isscontracts/mexico-7818-00.pdf

Forma en que soporta técnicamente al cliente que adquiere uno de los productos y/o servicios de donde extraemos la información pertinente para nuestro caso de investigación. (2017). https://technet.microsoft.com/esco/library/office-365-support.aspx

Políticas de Soporte Técnico de Software de Oracle. (2016). Oracle. https://www.oracle.com/lad/assets/softwarepolicy-lad.pdf

Formulario para Ayuda tecnológica. (2016). Solutek Informática. http://www.solutekcolombia.com/contacto/ay uda_tecnologica.htm 